\title{
Note on the structure of Kruskal's Algorithm
}

\author{
Nicolas Broutin Luc Devroye Erin McLeish*
}

November 15, 2007

\begin{abstract}
We study the merging process when Kruskal's algorithm is run with random graphs as inputs. Our aim is to analyze this process when the underlying graph is the complete graph on $n$ vertices lying in $[0,1]^{d}$, and edge set weighted with the Euclidean distance. The height of the binary tree explaining the merging process is proved to be $\Theta(n)$ on average. On the way to the proof, we obtain similar results for the complete graph and the $d$-dimensional square lattice with i.i.d. edge weights.
\end{abstract}

Keywords and phrases: Random trees, Minimum spanning tree, Kruskal, height, random graphs, percolation.

\section{Introduction}

The minimum spanning tree (MST) problem is one of the most studied in combinatorial optimization. In a connected graph $G=(V, E)$, each edge $e$ is given a weight $w(e)$. Let the weight of a graph be the sum of the weights of its edges. The MST problem consists then in computing a minimal weight tree, whose edges cover the entire set of vertices $V$. Fast greedy algorithms are known to solve this problem, namely the algorithms of Prim [10, 15, 25], Kruskal [18] and Borůvka [8]. Minimum spanning trees with random edge weights have been analyzed in several papers. Examples of such random graphs include the complete graph or $d$-dimensional hypercube with random independent edge weights [23], and the complete graph over $n$ points in $\mathbb{R}^{d}$ with the Euclidean distances as weights $[26,28]$. The properties studied include the overall weight [12], the degree sequence [2], and the maximum weight $[22,24]$.

In this paper, instead of studying the MST itself, we rather study the process that builds it. Such a point of view has already been taken by McDiarmid, Johnson, and Stone [19] who studied the first few steps of Prim's algorithm when the underlying graph is complete and the edge weights are independent identically distributed (i.i.d.) random variables. We give results for Kruskal's forest growing method, and more particularly about the structure of the merging process. With this in mind, we want to define a tree explaining Kruskal's algorithm, and we proceed as follows.

\footnotetext{
* Research of the authors was supported by NSERC Grant A3456 and by a James McGill Fellowship. Address: School of Computer Science, McGill University, Montreal H3A2K6, Canada. Email: \{nbrout, luc,mcleish\}@cs.mcgill.ca
} 
We grow two graph processes side by side. Assume that $G$ is the underlying weighted graph for which we are computing the MST. Let $\left\{M_{i}(G), 1 \leq i \leq n\right\}$ be the random graph process for Kruskal's algorithm on a graph $G$ of $n$ vertices, where $M_{i}(G)$ is the forest after exactly $i-1$ edges have been added, and $M_{n}(G)$ is the resulting MST. The second process is denoted by $\left\{F_{i}(G), i \leq i \leq n\right\}$, where $F_{i}(G)$ is a binary forest over $n+i-1$ vertices. We use $M_{i}$ and $F_{i}$ for short, when the underlying graph $G$ is already specified. The $F_{i}$ 's model the merging process between components during the execution of Kruskal's algorithm. Each tree in the forest $F_{i}$ is rooted, and each tree in $F_{i}$ corresponds to a connected component in $M_{i}$. One can think of the leaves of $F_{i}$ as the vertices in $M_{i}$ and the internal nodes as being the connecting edges. When $i=n$, the process finishes with the tree explaining Kruskal's algorithm: $T_{n} \stackrel{\text { def }}{=} F_{n}$. Also, let $\left\{\mathscr{F}_{i}, 1 \leq i \leq n\right\}$ be the natural filtration adapted to the random processes. The two processes grow from $i=1$ to $n$ in the following way: Initially, $M_{1}$ consists of $n$ isolated vertices $v_{1}, v_{2}, \ldots, v_{n}$ and no edges, and $F_{1}$ consists of $n$ vertices $u_{1}, u_{2}, \ldots, u_{n}$, which are merely copies of the vertices in the graph $G$. Kruskal's algorithm adds the edges of $G$ by increasing weight, as long as they do not create a cycle. Suppose we have already built $M_{i}$ and $F_{i}$, and that $e$ is the next edge to be added by Kruskal's algorithm. We grow the forest $M_{i+1}$ by simply adding the edge $e: M_{i+1}=M_{i} \cup\{e\}$. Next, suppose the endpoints of $e$ are vertices $u$ and $v$. Then the edge $e$ connects two components of $M_{i}$ : one containing vertex $u$, and the other, $v$. In the forest $F_{i}$, a component is uniquely represented by the root of the tree it corresponds to. Let $r_{u}$ and $r_{v}$ be the roots of the trees in $F_{i}$ containing $u$ and $v$ respectively. Grow the forest $F_{i+1}$ from $F_{i}$ by using two new edges and a new root to join the roots $r_{u}$ and $r_{v}$, creating one tree in $F_{i+1}$ out of the two trees in $F_{i}$. Eventually, the process leads to a random binary tree $T_{n}=F_{n}$ that models the way the components were merged during the execution of Kruskal's algorithm. The main parameter of interest for us is the height $H_{n}(G)$ of $T_{n}$, which is the height of the tree $T_{n}$ when the underlying weighted graph is $G$.

The purpose of the paper is to give asymptotic results for $H_{n}$ when the underlying graph is the complete graph over $n$ independent uniform points lying in $[0,1]^{d}$, and the edges are weighted with the Euclidean distance between their endpoints.

Theorem 1. Let $X_{1}, X_{2}, \ldots, X_{n}$ be $n$ independent uniform points in $[0,1]^{d}$. Let $E_{n}$ be the complete graph with vertex set $\left\{X_{1}, X_{2}, \ldots, X_{n}\right\}$. For each $1 \leq i, j \leq n$, let the weight of the edge $X_{i} X_{j}$ be the Euclidean distance between $X_{i}$ and $X_{j}$. Let $H_{n}\left(E_{n}\right)$ be the height of the tree $T_{n}\left(E_{n}\right)$ explaining Kruskal's algorithm when the input is $E_{n}$. Then there exists a constant $c \in(0,1)$ such that

$$
\lim _{n \rightarrow \infty} \mathbf{P}\left\{H_{n}\left(E_{n}\right) \geq c n\right\}=1 .
$$

In particular, $\mathbf{E} H_{n}\left(E_{n}\right)=\Theta(n)$, as $n$ goes to infinity.

Hence, in some sense, Kruskal's algorithm does not proceed very differently from Prim's algorithm, which grows a tree of height exactly $n-1$. Our proof uses ideas arising from two simpler examples. The Euclidean case has some dependence between the distances between points, and one can naturally consider first the complete graph $K_{n}$ with i.i.d. edge weights. 
Theorem 2. $H_{n}\left(K_{n}\right)$ is the height of the tree $T_{n}\left(K_{n}\right)$ explaining Kruskal's algorithm when the input is the complete graph $K_{n}$ with edges weighted by independent $[0,1]$ uniform random variables. There exists a constant $c \in(0,1)$ such that

$$
\lim _{n \rightarrow \infty} \mathbf{P}\left\{H_{n}\left(K_{n}\right) \geq c n\right\}=1 .
$$

As a consequence, $\mathbf{E} H_{n}=\Theta(n)$.

This simpler case is related to the analysis of union-find algorithms, and quickfind in particular [1]. In quickfind, the cost of merging two sets is proportional to the size of the one of them, the one that is "merged into" the other. A result of Knuth and Schönhage [17] about the average-case behavior in a model of Yao [27] shows that the cost of $n-1$ random unions is $\Theta\left(n^{2}\right)$. It immediately follows that some set of linear size must be involved in a linear number of union operations. Further information about quickfind and related algorithms may be found in the analysis of Bollobás and Simon [5, 6], which, as our proof of Theorem 2, relies on the theory of random graphs.

Further, we add a geometrical aspect to the previous example by considering a lattice. Let $\mathbb{L}^{d}$ be the $d$-dimensional square lattice: the infinite graph with vertex set $\mathbb{Z}^{d}$, and the edges between vertices at distance 1 from each other. Consider the graph $D_{n}$, which is the restriction of $\mathbb{L}^{d}$ to a $d$-dimensional cubic box consisting of $n$ vertices, where $n=k^{d}$ for some integer $k \geq 0$. Assign i.i.d. random weights to the edges in $D_{n}$.

Theorem 3. Let $H_{n}\left(D_{n}\right)$ be the height of the tree $T_{n}\left(D_{n}\right)$ explaining Kruskal's algorithm when the input is $D_{n}$ with edges weighted by independent $[0,1]$-uniform random variables. There exists a constant $c \in(0,1)$ such that

$$
\lim _{n \rightarrow \infty} \mathbf{P}\left\{H_{n}\left(D_{n}\right) \geq c n\right\}=1,
$$

as $n \rightarrow \infty$. Hence, in particular, $\mathbf{E} H_{n}\left(D_{n}\right)=\Theta(n)$.

After some remarks valid for all three cases (Section 2), we start by proving Theorems 3 and 2. Theorem 1 is proved in Section 5.

\section{Preliminaries}

The height $H_{n}$ of $T_{n}$ cannot exceed $n$, and it suffices to focus on a lower bound. The present section explains a generic strategy to obtain the required lower bound. Step $i$ of Kruskal's algorithm is the step when the $i$ th edge is added, i.e., $M_{i}$ becomes $M_{i+1}$. Observe that when a new edge is added to $M_{i}$, two components are joined together by the edge, and the two corresponding trees in $F_{i}$ are merged, using a new root and two new edges. After this merge, the height of the new tree in $F_{i+1}$ is at least one more than the height of either of the two trees before the merging. Suppose we track a particular component during the execution of Kruskal's algorithm. Let $C_{i}(x)$ be the connected component in $M_{i}$ containing vertex $x$. Each time this component is involved in a merge, the height of the corresponding tree in $F_{i}$ increases by at least 1. As a consequence, the number of merges that $C_{i}(x)$ is involved in, as $i$ increases to $n$, is a lower bound on the height of the final tree $T_{n}$. 
Let $\xi_{i}$ be the indicator random variable that $C_{i}(x) \neq C_{i+1}(x)$, in other words, that component $C_{i}(x)$ is merged to another component to create $C_{i+1}(x)$. Fix any $k, 1 \leq k \leq n-1$. Then the number of merges that the component $C_{k}(x)$ is involved in starting at time $k$ is exactly $\sum_{i=k}^{n-1} \xi_{i}$. The next lemma formalizes this as a lower bound for the height $H_{n}(G)$ for any graph $G$.

Lemma 1. Let $H_{n}(G)$ be the height of the tree explaining Kruskal's algorithm when run on the input graph $G$ with vertex set $V$. Fix some vertex $x \in V$. Define the indicator random variables $\xi_{i}, 1 \leq i \leq n-1$ to be 1 when the edge added at the ith step of Kruskal's algorithm is incident to the component containing vertex $x$. Then,

$$
H_{n}(G) \geq \sum_{j=k}^{n-1} \xi_{i}
$$

for $1 \leq k \leq n-1$.

Lemma 1 lies at the heart of the approach we use in Sections 3, 4 and 5 to find lower bounds on the height $H_{n}$ for the examples we study. In the following, we write $|\cdot|$ for the cardinality function. Unless specified otherwise, $|\cdot|$ counts vertices for components of graphs. Also, in Euclidean settings, we write $\|\cdot\|$ to denote the volume of a subset of $[0,1]^{d}$.

\section{Proof of Theorem 2}

In this section, we deal with the case of the complete graph weighted with i.i.d. $[0,1]$-uniform random variables. This version of the problem is tightly connected to the structure of Erdős-Rényi random graphs [11].

Observe first that the weights on the edges induce a random permutation of the edges. This permutation is a uniformly random permutation. In particular, the $m$ first edges of the permutation define a random graph $G_{n, m}$ consisting of $m$ random edges (see Janson et al. [14] or Bollobás [4]). Processing the elements of this random permutation in order, Kruskal's algorithm adds an edge to the spanning forest unless it creates a cycle. Accordingly, the connected components of $M_{i}\left(K_{n}\right)$ are the same (vertex-wise), as the connected components of the Erdős-Rényi random graph $G_{n, m}$ for some $m \geq i$. In other words, studying the structure of the merges in Kruskal's algorithm reduces to a similar study in a $G_{n, m}$ model. In their seminal paper [11], Erdös and Rényi showed that a phase transition occurs in the component structure of $G_{n, m}$ random graphs when $m=\lfloor\beta n / 2\rfloor$ and $\beta=1$. Indeed, for $\beta<1$, the largest component, $L_{1}$ of $G_{n,\lfloor\beta n / 2\rfloor}$ satisfies almost surely (a.s.) $\left|L_{1}\right|=\Theta(\log n)$, while for $\beta>1$ it has size $\Theta(n)$.

For our purposes, properties of $H_{n}\left(K_{n}\right)$ rely deeply on the component structure of $G_{n, m}$ : once a component is large enough, it tends to absorb more and more small components. Let $1<\beta<2$ and consider the random graph $G_{n, m}$ where $m=\lfloor\beta n / 2\rfloor$. The connected components of this graph correspond exactly to those in the forest $F_{m^{\prime}}$ for some $m^{\prime} \leq m$. Note that $m^{\prime}$ is a random variable. We consider the evolution of Kruskal's algorithm from this point on. Let $C_{m^{\prime}}$ be the largest component at time $m^{\prime}$, and let $C_{i}$ denote the component containing $C_{m^{\prime}}$ at a later 
step $i \in\left\{m^{\prime}, \ldots, n\right\}$ of the algorithm. Notice that the $C_{i}$ 's are increasing in size, that is, $\left|C_{i}\right| \leq\left|C_{i+1}\right|$ for any $i \in\left\{m^{\prime}, \ldots, n-1\right\}$. As in the previous section, let $\xi_{i}$ be the indicator random variable for the event that $C_{i}$ is merged with another component at time $i$. Then by Lemma 1

$$
H_{n}\left(K_{n}\right) \geq \sum_{i=m^{\prime}}^{n-1} \xi_{i}
$$

Now, for $\beta>1$, there is $\theta \in(0,1)$ such that the largest component of $G_{n,\lfloor\beta n / 2\rfloor}$ has at least $\theta n$ vertices with probability going to 1 . The constant $\theta$ is independent of $n$. Let $Z$ be the indicator random variable for the event that $\left|C_{m^{\prime}}\right| \geq \theta n$. Note that $\mathbf{P}\{Z=1\} \rightarrow 1$, as $n \rightarrow \infty$. Then certainly

$$
H_{n}\left(K_{n}\right) \geq \sum_{i=m^{\prime}}^{n-1} \xi_{i} \cdot Z
$$

Consider the forest $M_{i}$, and let $A_{i}$ be the set of edges in $K_{n}$ between $C_{i}$ and the rest of the graph. The edges in $A_{i}$ are the edges that may hook $C_{i}$ to an other component. Let $B_{i}$ be the remaining edges not incident to $C_{i}$ and which do not create cycles. Then

$$
\left|A_{i}\right| \geq\left(n-\left|C_{i}\right|\right) \cdot\left|C_{i}\right|
$$

and

$$
\left|B_{i}\right| \leq \frac{\left(n-\left|C_{i}\right|\right) \cdot\left(n-\left|C_{i}\right|-1\right)}{2} .
$$

The edge added at step $i$ is uniformly random among those not creating a cycle, and

$$
\mathbf{P}\left\{\xi_{i}=1|| C_{i} \mid\right\} \geq \frac{\left|A_{i}\right|}{\left|A_{i}\right|+\left|B_{i}\right|} \geq \frac{2\left|C_{i}\right|}{\left|C_{i}\right|+n} .
$$

The events $\xi_{i}=1, i \in\left\{m^{\prime}, \ldots, n-1\right\}$ are not independent, since the more often $C_{i}$ merges, the bigger it gets and the more likely it is to merge again. We define new random variables, completely independent of the graph process to go around this. Let $S_{i}$ be a Bernoulli random variable with parameter $\theta$. For $i \in\left\{m^{\prime}, \ldots, n-1\right\}$, $\xi_{i} \cdot Z$ stochastically dominates $S_{i} \cdot Z$ :

$$
\begin{aligned}
\mathbf{P}\left\{\xi_{i} \cdot Z=1\right\} & =\mathbf{P}\left\{\xi_{i}=1 \mid Z\right\} \cdot \mathbf{P}\{Z=1\} \\
& =\mathbf{P}\left\{\xi_{i}=1|Z,| C_{i} \mid \geq \theta n\right\} \cdot \mathbf{P}\{Z=1\} \\
& \geq \frac{2 \theta n}{2 n-1} \cdot \mathbf{P}\{Z=1\} \\
& \geq \theta \cdot \mathbf{P}\{Z=1\}=\mathbf{P}\left\{S_{i} \cdot Z=1\right\},
\end{aligned}
$$

where the last step follows from the independence of $S_{i}$ and $Z$. Thus, for every $t$,

$$
\mathbf{P}\left\{H_{n}\left(K_{n}\right) \geq t\right\} \geq \mathbf{P}\left\{\sum_{m^{\prime}}^{n-1} S_{i} \cdot Z \geq t\right\}=\mathbf{P}\left\{\sum_{m^{\prime}}^{n-1} S_{i} \geq t\right\} \cdot \mathbf{P}\{Z=1\} .
$$

Recall that $m^{\prime} \leq n \beta / 2$. By the law of large numbers,

$$
\mathbf{P}\left\{\sum_{m^{\prime}}^{n-1} S_{i} \geq n\left(1-\frac{\beta}{2}\right) \cdot \frac{\theta}{2}\right\} \geq \mathbf{P}\left\{\sum_{m^{\prime}}^{n-1} S_{i} \geq\left(n-m^{\prime}\right) \cdot \frac{\theta}{2}\right\} \underset{n \rightarrow \infty}{\longrightarrow} 1 .
$$


Finally, $Z \rightarrow 1$ almost surely, and

$$
\mathbf{P}\left\{H_{n}\left(K_{n}\right) \geq n \cdot \frac{\theta}{2} \cdot\left(1-\frac{\beta}{2}\right)\right\} \underset{n \rightarrow \infty}{\longrightarrow} 1 .
$$

Since, $\theta(1-\beta / 2)>0$ by our definitions of $\beta$ and $\theta$, this completes the proof of Theorem 2.

\section{Proof of Theorem 3}

We now deal with the case of the finite box $D_{n}$ in the cubic lattice $\mathbb{L}^{d}$. As in the proof of Theorem 2, we show that there is a large component at some intermediate stage of Kruskal's algorithm, which will be merged to a linear number of other components as the algorithm goes on. By Lemma 1, this gives a lower bound on $H_{n}\left(D_{n}\right)$. To ensure a linear number of merges, we look for a large component with a specified structure involving "traps" for other components. Our approach is constructive and goes in two phases. First, a connected component $C_{p_{0}}$ made of a large number of traps is built using only edges of weight less than a fixed constant $p_{0} \in(0,1)$. The proof of its existence relies on concepts from percolation theory $[13,16]$. Then, the minimal spanning tree process is completed by adding the edges with weight more than $p_{0}$. In this second phase, the traps prevent small components from merging together before they hook up to $C_{p_{0}}$, hence the large number of merges to $C_{p_{0}}$.

We begin by defining a subset of $V\left(D_{n}\right)$, denoted $I_{n}$, for the sites of a percolation process. Suppose that the left bottom corner vertex of $D_{n}$ is at the origin, and using this coordinate system, let

$$
I_{n}=\left\{v: \forall i, v_{i} \bmod 4=1\right\},
$$

where $v_{j}$ is the $j$ th coordinate of vertex $v$. Each $x \in I_{n}$ is a site in a (site) percolation process. Two sites in $u, v \in I_{n}$ are neighbors if they are at distance 4 from each other along any one coordinate, i.e., $u$ and $v$ are neighbors if $|u-v|_{1}=4$ and $|u-v|_{\infty}=4$. For any $x \in I_{n}$, define a structural event $E_{x}$ which is depicted in Figure 1, and defined in terms of the following sets:

$$
U(x)=\left\{v \in V\left(D_{n}\right):|v-x|_{\infty}=1\right\},
$$

where $|\cdot|_{\infty}$ defines the $\ell_{\infty}$ - norm. Next, we define the edges on the unit box around $x$ :

$$
N_{1}(x)=\left\{e=(u, v): u, v \in U(x),|u-v|_{1}=1\right\} .
$$

Define the sets

$$
F_{1}(x)=\left\{v \in V\left(D_{n}\right):|v-x|_{1}=1\right\}
$$

and

$$
F_{2}(x)=\left\{v \in V\left(D_{n}\right):|v-x|_{1}=2 \text { and }|v-x|_{\infty}=2\right\},
$$

where $|\cdot|_{1}$ defines the $\ell_{1}$-norm. Finally, the outward edges in Figure 1 are defined as:

$$
N_{2}(x)=\left\{e=(u, v): u \in F_{1}(x) \text { and } v \in F_{2}(x)\right\} .
$$




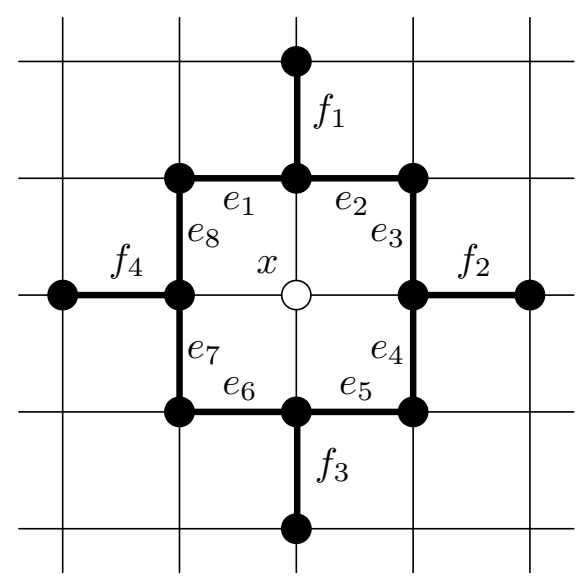

Figure 1. A portion of the square lattice $\mathbb{L}^{2}$ is shown, and the event $E_{x}$. Edges labelled $e_{1}, \ldots, e_{8}$ are in $N_{1}(x)$, and edges labelled $f_{1}, \ldots, f_{4}$ are in $N_{2}(x)$.

Figure 2. An instance of site percolation on $I_{n}$, where the probability of an open site $x$ is exactly the probability that event $E_{x}$ occurs.

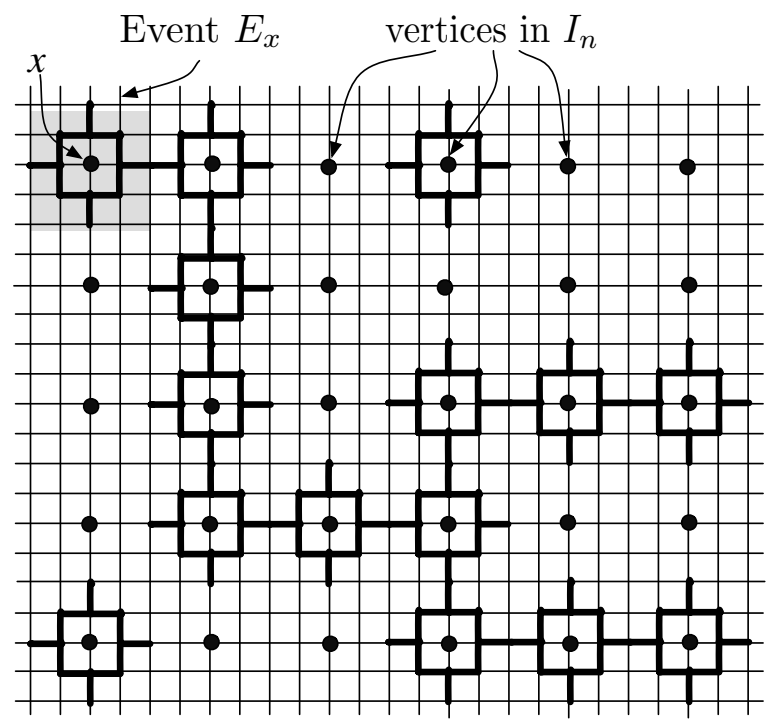

The event $E_{x}$ holds when all edges in $N_{1}(x)$ and $N_{2}(x)$ have weight less than $p_{0}$. Figure 1 depicts the event $E_{x}$ in $d=2$. Note that there is no restriction on the weights of the other edges (those not in $N_{1}(x)$ or $N_{2}(x)$ ) in the event $E_{x}$.

A site $x \in I_{n}$ is declared open if the event $E_{x}$ is true. This defines precisely a site percolation process since the probability that sites are open are independent from site to site. Figure 2 shows an instance of this site percolation process. A cluster in this model is defined in the usual way: the cluster of a site $x$ is the set of all open sites that can be reached along paths of open sites from $x$. For example, Figure 2 shows a cluster of size 13. Each site is a "trap" for its center vertex, and the goal is now to show the existence of a large open cluster in the site percolation process for some choice of $p_{0} \in(0,1)$.

It has been known for quite some time that the usual site percolation process on the infinite lattice $\mathbb{L}^{d}$ exhibits a phase transition phenomenon analogous to that in the random graph process: there exists a constant $p_{c} \in(0,1)$ such that for $p<p_{c}$, the is almost surely no infinite cluster, whereas for $p>p_{c}$ such an infinite cluster a.s. exists $[13,16]$. In our model, we are dealing with site percolation on a finite 
lattice, which is in fact a subgraph of the infinite lattice $\mathbb{L}^{d}$. The phase transition on the finite lattice has been analyzed by Borgs, Chayes, Kesten, and Spencer [7], who obtained an exact analog of the phase transition in random graphs for bond percolation in the finite hypercube $\Lambda_{n}=[0, n)^{d}$. In particular, they showed:

Lemma 2. Let $\Lambda_{n}$ denote a hypercube lattice of size $n$. Consider bond percolation, where each edge in the lattice is open with probability $p$, and let $L_{1}^{\text {bond }}(p)$ be the size of the largest open cluster in this hypercube. Let $\left|C_{p}(0)\right|$ denote the size of the open cluster at the origin on the infinite lattice $\mathbb{L}^{d}$, and let $\theta^{\text {bond }}(p)=\mathbf{P}\left\{\left|C_{p}(0)\right|=\infty\right\}$. Finally, let $p_{c}^{\text {bond }}$ be the critical probability for an infinite cluster in bond percolation on the infinite lattice $\mathbb{L}^{d}$. Then for all $p>p_{c}^{\text {bond }}$,

$$
\frac{\left|L_{1}^{\text {bond }}(p)\right|}{\left|\Lambda_{n}\right| \cdot \theta^{\text {bond }}(p)} \underset{n \rightarrow \infty}{\longrightarrow} 1 \quad \text { in probability. }
$$

A simple extension of this lemma is the following corollary, which gives a lower bound on the height of the largest open cluster in site percolation. The proof of the lemma is included at the end of this section.

Lemma 3. Consider site percolation on the finite hypercube lattice $\Lambda_{n}$. Let $L_{1}^{\text {site }}(p)$ be the largest open cluster. There exist constants $c_{1}>0$ and $p_{s} \in(0,1)$ such that for $p>p_{s}$ :

$$
\mathbf{P}\left\{\left|L_{1}^{\text {site }}(p)\right| \geq c_{1} n^{d}\right\} \underset{n \rightarrow \infty}{\longrightarrow} 1 .
$$

The constants $c_{1}$ and $p_{s}$ depend only on the dimension $d$.

In our site percolation model, the probability that a site is open is exactly $\mathbf{P}\left\{E_{x}\right\}$, which depends on our choice of $p_{0}$. We are now ready to nail down the constant $p_{0}$. With the previous definitions of $N_{1}(x)$ and $N_{2}(x), \mathbf{P}\left\{E_{x}\right\}=p_{0}^{\left|N_{1}(x)\right|+\left|N_{2}(x)\right|}$. The sizes of the sets $N_{1}(x)$ and $N_{2}(x)$ are increasing in $d$, but constant in terms of $n$. Therefore, we can choose $p_{0} \in(0,1)$ large enough that $p_{s}<\mathbf{P}\left\{E_{x}\right\}<1$, where $\mathbf{P}\left\{E_{x}\right\}$ is a function of $d$ only. Consider now the largest open cluster in the site percolation process on $I_{n}$ and let $L_{1}\left(I_{n}\right)$ be its vertex set. Note that by definition, the lattice $I_{n}$ has size $\left|I_{n}\right| \geq n / 4^{d}$. By Lemma 3 ,

$$
\left.\mathbf{P}\left\{\mid L_{1}\left(I_{n}\right)\right) \mid \geq \frac{c_{1} n}{4^{d}}\right\} \underset{n \rightarrow \infty}{\longrightarrow} 1 .
$$

Next, we isolate the vertices of the connected component of $D_{n}$ associated with the largest open cluster, $L_{1}\left(I_{n}\right)$ :

$$
C_{p_{0}}=\left\{v \in V\left(D_{n}\right): \exists x \in L_{1}\left(I_{n}\right), v \in U(x) \text { or } v \in F_{2}(x)\right\},
$$

Recall that $\left\{M_{i}, 1 \leq i \leq n\right\}$ is the graph process associated with Kruskal's algorithm, and let $i^{\star}$ be the largest index for which all the edges of $M_{i^{\star}}$ have weight at most $p_{0}$. Then the vertices of $C_{p_{0}}$ belong to a connected component in $M_{i^{\star}}$. Thus it remains only to provide a lower bound on the number of merges to this component as Kruskal's algorithm completes. 
For each $x \in I_{n}$, we define an event $J_{x}$ which ensures that the "trap" at $x$ is successful and that a small component joins $C_{p_{0}}$ at some stage $p>p_{0}$ of Kruskal's algorithm. Define the set of edges

$$
N_{3}(x)=\left\{e=(x, v):|v-x|_{1}=1\right\} .
$$

Then the event $J_{x}$ is the event that all edges in $N_{3}(x)$ have weight more than $p_{0}$. Thus if $J_{x}$ holds, the vertex $x$ is not connected to any vertex in $C_{p_{0}}$ in the graph $M_{i^{\star}}$, but it will be connected at some later stage of Kruskal's algorithm. Notice that $J_{x}, x \in I_{n}$, are defined using edges disjoint from $N_{1}(y)$ and $N_{2}(y)$, for $y \in I_{n}$, so that $\left\{J_{x}, x \in I_{n}\right\}$ is independent of $\left\{E_{y}, y \in I_{n}\right\}$. Moreover, the events $J_{x}, x \in I_{n}$, are independent. The proof of the theorem is now straightforward: we show that $J_{x}$ is true for a constant proportion of the sites in $L_{1}\left(I_{n}\right)$, implying that a constant proportion of sites in $L_{1}\left(I_{n}\right)$ will be responsible for merges to that component as Kruskal completes. In other words, by Lemma 1,

$$
H_{n}\left(D_{n}\right) \geq \sum_{x \in L_{1}\left(I_{n}\right)} \mathbf{1}\left[J_{x}\right]
$$

But the events $J_{x}$ and $\left\{x \in L_{1}\left(I_{n}\right)\right\}$ are independent, and therefore by (2),

$$
\mathbf{P}\left\{\sum_{x \in L_{1}\left(I_{n}\right)} \mathbf{1}\left[J_{x}\right] \geq c n\right\} \underset{n \rightarrow \infty}{\longrightarrow} 1
$$

where $c=c_{1}(1-p)^{2 d} /\left(2 \cdot 4^{d}\right)$. It follows that $\mathbf{P}\left\{H_{n}\left(D_{n}\right) \geq c n\right\} \rightarrow 1$ as $n \rightarrow \infty$, which completes the proof of Theorem 3 .

Proof of Lemma 3. Consider the lattice $\Lambda_{n}$ where each edge $e=(x, y)$ is represented by two coloured edges, one red and one green. Each coloured edge is opened independently with probability $p$. Now consider a bond percolation process on $\Lambda_{n}$ where a bond is considered open if both the red and green edges between two vertices are open. Thus the bond probability is exactly $p^{2}$. Let $L_{1}^{\text {bond }}\left(p^{2}\right)$ be the largest open cluster in this bond percolation process on $\Lambda_{n}$.

Next, consider a site percolation process, also on $\Lambda_{n}$. Begin by duplicating the edges as described above: each edge is replaced by one red and one green edge, and each coloured edge is opened with probability $p$. Next, alternate colouring the vertices of $\Lambda_{n}$ red and green, so a red vertex is surrounded by green vertices, and vice versa. A red site is declared open if at least one of its incident red edges is open. A green site is open if at least one of its incident green edges is open. Because of the alternating colours, sites are opened independently, with probability $1-(1-p)^{2 d}$. An open cluster is defined in the usual way to be a cluster of open sites, regardless of their colour. Let $L_{1}^{\text {site }}\left(p^{\prime}\right)$ be the size of the largest cluster in this site percolation process on $\Lambda_{n}$, where $p^{\prime}=1-(1-p)^{2 d}$.

Now we couple the two models. Consider and instance of $\Lambda_{n}$ with duplicated edges, where we open the coloured edges with probability $p$. Notice that if a bond is open in the bond percolation model, then both the red and green edges are open. So the red vertex incident to the red edge is open in the site model, and the green vertex incident to the green edge is also open in the site model. Thus the two 
neighboring sites are both open in the site model. This implies that the vertices in the cluster $L^{\text {bond }}\left(p^{2}\right)$ are also in a cluster in the site percolation model. Thus $\left|L^{\text {bond }}\left(p^{2}\right)\right| \leq\left|L^{\text {site }}\left(p^{\prime}\right)\right|$. By Lemma 2 , if $p^{2}>p_{c}^{\text {bond }}$, then $\mathbf{P}\left\{\left|L_{1}^{\text {bond }}\left(p^{2}\right)\right| \geq c_{1} n^{d}\right\} \rightarrow$ 1 for a constant $c_{1}>0$. Thus

$$
\mathbf{P}\left\{\left|L_{1}^{\text {site }}\left(1-(1-p)^{2 d}\right)\right| \geq c_{1} n^{d}\right\} \underset{n \rightarrow \infty}{\longrightarrow} 1
$$

for $p^{2}>p_{c}^{\text {bond }}$. So in general, for any $p>1-\left(1-\sqrt{p_{c}^{\text {bond }}}\right)^{2 d}$,

$$
\mathbf{P}\left\{\mid L_{1}^{\text {site }}(p) \geq c_{1} n^{d}\right\} \underset{n \rightarrow \infty}{\longrightarrow} 1 .
$$

Setting $p_{s}=1-\left(1-\sqrt{p_{c}^{b o n d}}\right)^{2 d} \in(0,1)$ completes the proof.

\section{$5 \quad$ Random Euclidean edge lengths}

Random Euclidean minimum spanning trees are related to random geometric graphs [21] and continuum percolation [20]. One can define an analog of Erdős-Renyi random graphs $[4,14]$ where the vertex set consists of points lying in the cube $[0,1]^{d}$. The distance between pairs of vertices is defined to be the Euclidean distance. The classical coupling used to build Erdős-Rényi $G_{n, p}$, assigns to each edge of the complete graph $K_{n}$ an independent copy of a [0,1]-uniform random variable, and lets $G_{n, p}$ be the graph containing edges shorter than $p$. Similarly, the random geometric graph $G\left(\mathcal{X}_{n}, r\right)$ is defined to be the graph with vertex set $\mathcal{X}_{n}$, which consist of $n$ independent uniform points in $[0,1]^{d}$, and edges shorter than $r$. For technical reasons, it is usually easier to consider random graphs $G\left(\mathcal{P}_{n}, r\right)$ that are defined similarly, but with vertex set given by a Poisson point process $\mathcal{P}_{n}$ with rate $n$ in $[0,1]^{d}$. It is convenient to see random geometric graphs as a vertex set $\mathcal{X}$ together with closed balls $B(x, r / 2)$ of radius $r / 2$ surrounding every $x \in \mathcal{X}$. A pair of points $x, y \in \mathcal{X}$ share an edge in $G(\mathcal{X}, r)$ if and only if $B(x, r / 2)$ and $B(y, r / 2)$ intersect. Equivalently, the points $x, y \in \mathcal{X}$ share an edge if the Euclidean distance from $x$ to $y$ is at most $r$.

The graphs $G\left(\mathcal{X}_{n}, r_{n}\right)$ and $G\left(\mathcal{P}_{n}, r_{n}\right)$ with radius

$$
r_{n}=\left(\frac{\lambda}{n}\right)^{1 / d}
$$

are known to admit a threshold for the size of the largest component for some critical $\lambda_{c}>0$ [20]. In other words, if $L_{1}(n)$ is the largest component in the random graph $G\left(\mathcal{P}_{n}, r_{n}\right)$ in $[0,1]^{d}$, then there exists a $\lambda_{c}$ such that if $\lambda>\lambda_{c}$,

$$
n^{-d}\left|L_{1}(n)\right| \underset{n \rightarrow \infty}{\longrightarrow} \lambda p_{\infty}(\lambda),
$$

in probability [21, Theorem 10.9]. So, as in the $G_{n, p}$ model, the largest component has size linear in the volume. The constant $p_{\infty}(\lambda)$ is defined in terms of a Poisson process $\mathcal{P}$ with rate 1 in $\mathbb{R}^{d}$ : it is the probability that the origin lies in the set of balls of an infinite component of the geometric graph $G\left(\mathcal{P}, \lambda^{1 / d}\right)$. Random geometric graphs differ from the Erdős-Rényi model when we consider the second largest 
component. For the Erdős-Rényi random graph with $p=c n / 2$ and $c>1$, the order of the second largest component grows as $\log n$. For the random geometric graph, it grows not faster than a larger power of the logarithm [21, Theorem 10.18]. Let $L_{2}(n)$ be the size of the second largest component in $G\left(\mathcal{P}_{n}, r_{n}\right)$ and $\lambda>\lambda_{c}$, then for $d \geq 2$, there exists a constant $c_{1}$ such that

$$
\mathbf{P}\left\{L_{2}(n)<c_{1} \cdot(\log n)^{d /(d-1)}\right\} \underset{n \rightarrow \infty}{\longrightarrow} 1 .
$$

With these two facts in hand, a lower bound on the height $H_{n}$ immediately follows. We can grow the minimum spanning forest using increasing radii $r_{n}=$ $(\lambda / n)^{1 / d}$, until $\lambda>\lambda_{c}$. Then, use the number of components that hook up to $L_{1}(n)$ from this point on as a lower bound on $H_{n}$. The size of any component merging with $L_{1}(n)$ is at most of order $(\log n)^{d /(d-1)}$ and hence

$$
H_{n}=\Omega\left(\frac{n}{(\log n)^{d /(d-1)}}\right)
$$

in probability. We now strengthen this bound. We begin by proving the following Poissonized version of Theorem 1 . Theorem 1 is proved for a fixed number of points in Section 6.

Theorem 4. Let $N$ be a Poisson random variable with mean $n$. Let $X_{1}, X_{2}, \ldots, X_{N}$ be $N$ independent uniform points in $[0,1]^{d}$. Let $E_{N}$ be the complete graph with vertex set $\left\{X_{1}, \ldots, X_{N}\right\}$ and edge weights given by the Euclidean distance between $X_{i}$ and $X_{j}$. Then, the height of the tree explaining Kruskal's algorithm with input $E_{N}$ satisfies

$$
\lim _{n \rightarrow \infty} \mathbf{P}\left\{H_{N}\left(E_{N}\right) \geq c n\right\}=1,
$$

for some $c \in(0,1)$. Also, $\mathbf{E} H_{N}\left(E_{N}\right)=\Theta(n)$, as $n$ goes to infinity.

The proof of the above theorem relies on techniques similar to the ones we developed in the proof of Theorem 3. Consider the forest $M_{i^{\star}}$ produced by Kruskal's algorithm at step $i^{\star} \in\{1, \ldots, n-1\}$, the largest $i$ such that all edges of $M_{i}$ have weights less than $r_{n}=\left(\lambda_{0} / n\right)^{1 / d}$, for a fixed constant $\lambda_{0}>0$ to be chosen later. We prove the existence of a large component consisting of small "traps" with certain good properties, and count the number of new components that join the large one.

The analysis becomes easier if we rescale to a box of volume $n$. Instead of a Poisson process with rate $n$, consider a Poisson point process with rate 1 in the cubic box $\left[0, n^{1 / d}\right]^{d}$ of volume $n$. Note that for this rescaled process, the radius of the balls is $\lambda^{1 / d}$ instead of $(\lambda / n)^{1 / d}$. We refer to this graph process as $G(\mathcal{P}, r)$, where now $\mathcal{P}$ is the set of Poisson points in $\left[0, n^{1 / d}\right]^{d}$, and $r=\lambda^{1 / d}$.

Define

$$
t=\left\lfloor\frac{n^{1 / d}}{\alpha \lambda_{0}^{1 / d}}\right\rfloor,
$$

for a constant $\alpha>0$, and partition $\left[0, n^{1 / d}\right]^{d}$ into exactly $t^{d}$ smaller cubic boxes each of side length $\alpha \lambda_{0}^{1 / d}\left(1+O\left(n^{-1 / d}\right)\right)$. Label these smaller boxes $Q_{1}, Q_{2}, \ldots, Q_{t^{d}}$. The partition is shown in Figure 3. 


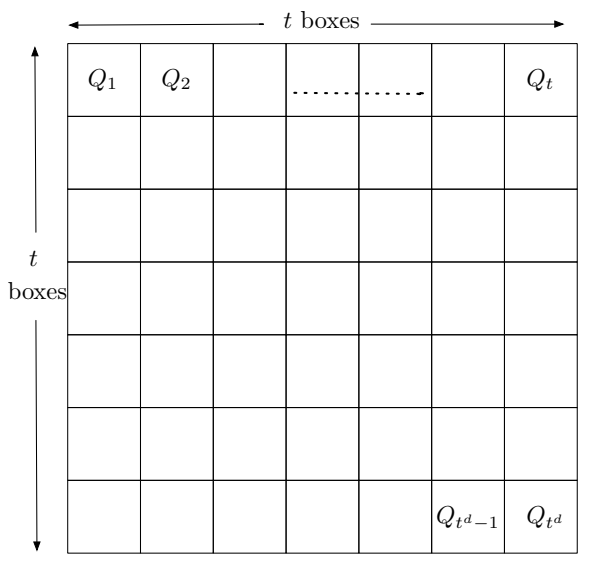

Figure 3. The region $\left[0, n^{1 / d}\right]^{d}$ and its division into $t^{d}$ boxes, labeled $Q_{1}, \ldots, Q_{t^{d}}$

We start by describing particular events $E_{i}, 1 \leq i \leq t^{d}$, where each $E_{i}$ depends only on the points lying in $Q_{i}$. In essence, $E_{i}$ is the event that the boundary of $Q_{i}$ is "covered" by balls of radius $\lambda_{0}$ centered at the poisson points. This is illustrated in Figure 3. Each box $Q_{i}$ is further split into even smaller boxes, $B_{i, j}, 1 \leq j \leq\lceil\alpha \sqrt{d}\rceil^{d}$ (see Figure 4). Each box $B_{i, j}$ has side length $\ell$ such that

$$
\left(1-n^{-1 / d}\right) \lambda_{0}^{1 / d} / \sqrt{d} \leq \ell \leq \lambda_{0}^{1 / d} / \sqrt{d} .
$$

Let $S_{i}$ be the set of indices $j$ for which $B_{i, j}$ touches the boundary of $Q_{i}$. Each box $B_{i, j}, j \in S_{i}$ has a kernel $K_{i, j}$, which is a box located at the center of $B_{i, j}$. The kernel boxes $K_{i, j}$ are chosen with side length $\ell / 3$. The event $E_{i}$ is the event that there is at least one point in each $K_{i, j}, j \in S_{i}$ :

$$
E_{i}=\bigcap_{j \in S_{i}}\left\{\mathcal{P} \cap K_{i, j} \neq \emptyset\right\}
$$

From now on, we refer to the boundary of $Q_{i}$ as the region $\bigcup_{j \in S_{i}} B_{i, j}$.

Let us first verify that the boundary boxes $B_{i, j}, j \in S_{i}$ are connected in $Q_{i}$. Suppose $x \in K_{i, j}, j \in S_{i}$. The distance from $x$ to a corner point of the box is at most:

$$
(2 / 3) \ell \sqrt{d} \leq(2 / 3) \lambda_{0}^{1 / d},
$$

where we upper bound $\ell$ using (3). But the radius of the ball centered at $x$ is $r=\lambda_{0}^{1 / d}$, implying that $B(x, r)$ will contain all corners of $B_{i, j}$. Therefore if $B_{i, j}$ and $B_{i, k}$ are neighbors and $x \in K_{i, j}, y \in K_{i, k}$, then the balls $B(x, r)$ and $B(y, r)$ intersect. Hence if $E_{i}$ occurs, all points in the $B_{i, j}, j \in S_{i}$ are part of the same connected component in $G(\mathcal{P}, r)$. Notice also that if boxes $Q_{i_{1}}$ and $Q_{i_{2}}$ are neighbors in the partition of Figure 3 and if $E_{i_{1}}$ and $E_{i_{2}}$ both hold, then all the points in the boundaries of each box lie in the same connected component of $G(\mathcal{P}, r)$.

Now let each box $Q_{i}, 1 \leq i \leq t^{d}$, be a site in a (site) percolation process. A site is declared open if the event $E_{i}$ holds in the corresponding box $Q_{i}$. Since the events $E_{i}$ are independent and occur with the same probability, this defines a site percolation process on the finite square lattice of $t^{d}$ sites. Figure 5 shows a portion of the site percolation process. An open site creates the "trap" we are looking for, since the points lying in the inside have to merge with the boundary. 

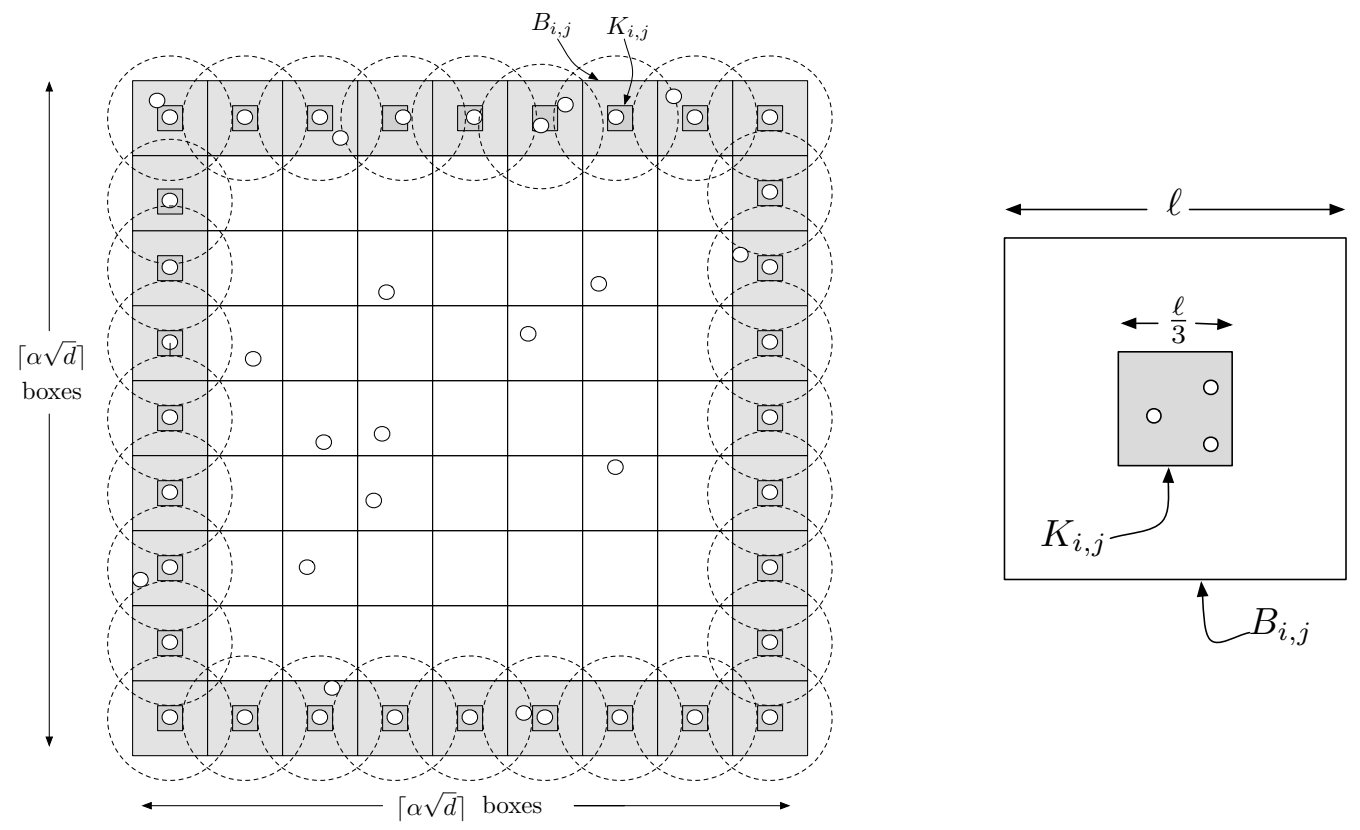

Figure 4. The box $Q_{i}$ when the event $E_{i}$ is true. The shaded region represents the boundary region $S_{i}$ of $Q_{i}$. The kernel boxes $K_{i, j}$ are shown, and the ball of radius $\lambda_{0}^{1 / d}$ centered at a point in $K_{i, j}$. The figure also shows a close-up of a small box $B_{i, j}$, and its kernel box, $K_{i, j}$.

The probability $\mathbf{P}\left\{E_{i}\right\}$ that a specified site is open is a constant depending only on $\lambda_{0}$ and $d$. More specifically, the probability that there is at least one point in a $K_{i_{j}}$ is at least $1-\exp \left(-(\ell / 3)^{d}\right),\left|S_{i}\right| \leq 2 d(\alpha \sqrt{d}+1)$. Thus

$$
\mathbf{P}\left\{E_{i}\right\} \geq\left(1-\exp \left(-(\ell / 3)^{d}\right)\right)^{\left|S_{i}\right|} .
$$

We are again interested in the existence of a large component of open sites. By Lemma 3, there exists a $p_{s}>0$ such that if the probability of an open site is $p$, and $p>p_{s}$, then $\mathbf{P}\left\{\left|L_{1}^{\text {site }}(p)\right| \geq c_{1} t^{d}\right\} \rightarrow 1$, as $t \rightarrow \infty$. Thus in order to guarantee that $\mathbf{P}\left\{E_{i}\right\}>p_{s}$, we require that

$$
-\log \left(1-p_{s}^{1 /\left|S_{i}\right|}\right)<(\ell / 3)^{d} .
$$

By definition, $\ell \geq\left(1-n^{-1 / d}\right) \lambda_{0}^{1 / d} / \sqrt{d}$, and so $\ell \geq \lambda_{0}^{1 / d} /(2 \sqrt{d})$ for $n>2$. Notice the left side of Equation (4) is a constant, thus we can choose $\lambda_{0}>0$ large enough that satisfies (4). Let $L_{1}\left(I_{n}\right) \subseteq\left\{1, \ldots, t^{d}\right\}$ denote the index set of the sites in the largest open component. Then by Lemma 3 ,

$$
\mathbf{P}\left\{\left|L_{1}\left(I_{n}\right)\right| \geq c_{1} t^{d}\right\} \underset{n \rightarrow \infty}{\longrightarrow} 1 .
$$

Next, we establish the existence of a large connected component in the random geometric graph. Let

$$
C_{\lambda_{0}}=\left\{x \in \mathcal{P}: x \in \cup_{j \in S_{i}} B_{i, j}, \text { for some } i \in L_{1}\left(I_{n}\right)\right\},
$$

then the vertices in $C_{\lambda_{0}}$ belong to a connected component in $G\left(\mathcal{P}, \lambda_{0}\right)$. It remains only to provide a lower bound on the number of merges to that connected component as Kruskal's algorithm completes (i.e., as $\lambda$ increases above $\lambda_{0}$ ). 


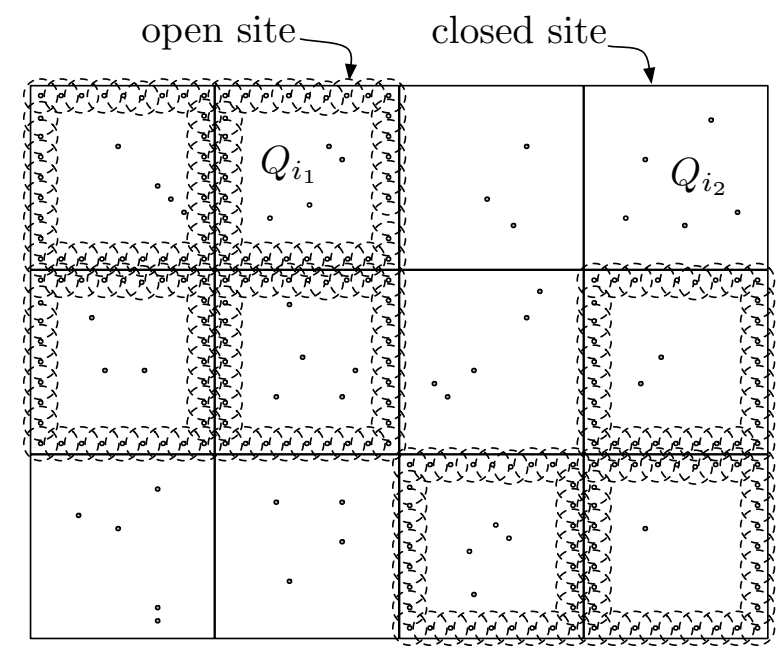

Figure 5. A portion of the site percolation process on sites $Q_{i}, i \in\left\{1, \ldots, t^{d}\right\}$. Notice that when two open sites are neighbours, then the vertices in the boundaries of each site are in the same connected component in $G\left(\mathcal{P}, \lambda_{0}\right)$.

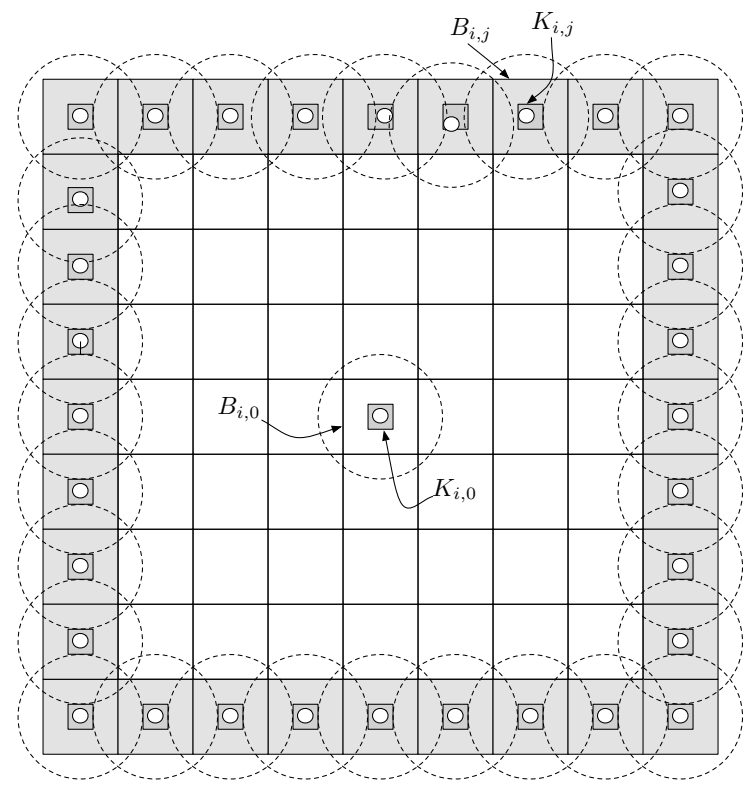

Figure 6. Event $J_{i}$ for the box $Q_{i}$. The only Poisson points are in $K_{i, 0}$ and $K_{i, j}$ for $j \in S_{i}$.

Define a new event $J_{i}$ depending only on the points in $Q_{i}$ in the following way: suppose $B_{i, 0}$ is the mini-box at the very center of $Q_{i}$. Let $K_{i, 0}$ be the kernel box associated with this $B_{i, 0}$. The event $J_{i}$ occurs when there is at least one point in $K_{i, 0}$ and the rest of $Q_{i}$ is free of points, except for the boundary region. In other words,

$$
J_{i}=\left[\mathcal{P} \cap\left\{B_{i_{0}} \backslash K_{i_{0}}\right\}=\emptyset\right] \cap\left[\mathcal{P} \cap K_{i_{0}} \neq \emptyset\right] \cap\left[\bigcap_{j \notin S_{i}, j \neq 0}\left\{\mathcal{P} \cap B_{i_{j}}=\emptyset\right\}\right] .
$$

The event $J_{i}$ is illustrated in Figure 6, and ensures that the "trap" created by $E_{i}$ is successful. As with the event $E_{i}, \mathbf{P}\left\{J_{i}\right\}>0$ is a constant depending only on $\lambda_{0}$ and $d$. Clearly, the events $J_{i}$ are independent from each other and from all the events $E_{i}$.

Let us first show that at $r=\lambda_{0}^{1 / d}$ any points in $K_{i, 0}$ are not connected to the boundary points. Set $\alpha=6$, let $x \in K_{i, 0}$ and suppose $J_{i}$ is true. Observe that the 
distance from $x$ to any other Poisson point in $Q_{i}$ (but outside of $K_{i, 0}$ ) is at least

$$
(\alpha \sqrt{d} / 2-2) \ell=(3-2 / \sqrt{d})\left(1-n^{-1 / d}\right) \lambda_{0}^{1 / d} \geq 3 \lambda_{0}^{1 / d}\left(1-n^{-1 / d}\right)>2 \lambda_{0}^{1 / d}=2 r,
$$

for $n^{1 / d}>3$. Therefore, the ball centered at $x$ does not intersect any other balls in $Q_{i}$, if $n^{1 / d}>3$. In other words, $x \in K_{i, 0}$ is not connected to the vertices of $C_{\lambda_{0}}$ in the graph $M_{i^{\star}}$, but it will be at some later stage of Kruskal's algorithm.

Finally, let $M(n)$ be the number of merges to the vertices of $C_{\lambda_{0}}$ for $i>i^{\star}$. Each event $J_{i}$ accounts for one connection to $C_{\lambda_{0}}$, thus

$$
M(n) \geq \sum_{i \in L_{1}\left(I_{n}\right)} J_{i}
$$

Using the fact that $\mathbf{P}\left\{\left|L_{1}\left(I_{n}\right)\right| \geq c_{1} t^{d}\right\} \rightarrow 1$ and that $\mathbf{P}\left\{J_{i}\right\}>0$ is constant, where $J_{i}$ is independent of the event $\left\{i \in L_{1}\left(I_{n}\right)\right\}$, we conclude that

$$
\mathbf{P}\left\{M(n) \geq\left(c_{1} / 2\right) \mathbf{P}\left\{J_{i}\right\} t^{d}\right\} \underset{n \rightarrow \infty}{\longrightarrow} 1 .
$$

Now use the definition of $t$ and:

$$
\mathbf{P}\{M(n) \geq c n\} \underset{n \rightarrow \infty}{\longrightarrow} 1,
$$

where $c=c_{1} \mathbf{P}\left\{J_{i}\right\} /\left(2 \alpha^{d} \lambda_{0}\right)$ is a constant independent of $n$. This finishes the proof of Theorem 4 .

\section{Proof of Theorem 1}

In this section, we extend the result of the previous section for a fixed number of points uniformly distributed in $[0,1]^{d}$. In other words, we depoissonize Theorem 4 to prove Theorem 1. From now on, we use $H_{n}$ and $T_{n}$ instead of $H_{n}\left(E_{n}\right)$ and $T_{n}\left(E_{n}\right)$, since we are always referring to the graph $E_{n}$ (defined in Section 1), constructed using a point set $\mathcal{X}_{n}=\left\{x_{1}, \ldots, x_{n}\right\}$ where each $x_{i} \in[0,1]^{d}$.

Lemma 4 (Stability of the Euclidean MST). Let $x_{1}, x_{2}, \ldots, x_{n+1} \in[0,1]^{d}$. Let $M S T_{n}$ and $M S T_{n+1}$ be the minimum spanning trees on $\mathcal{X}_{n}$ and $\mathcal{X}_{n+1}$, respectively, where Euclidean distance is used for the edge weights. Assume both $M S T_{n}$ and $M S T_{n+1}$ are unique. Then $M S T_{n}$ and $M S T_{n+1}$ differ by at most $2 \operatorname{deg}\left(x_{n+1}\right)=$ $O(1)$ edges, where $\operatorname{deg}(\cdot)$ denotes the degree in $M S T_{n+1}$.

Proof. Let $N$ be the set of neighbours of $x_{n+1}$ in $M S T_{n+1}$. We can build $M S T_{n+1}$ from $M S T_{n}$ as follows: Add vertex $x_{n+1}$ and add all edges from $x_{n+1}$ to its neighbors $y \in N$. Next, we use the well known characterization of edges of the minimum spanning tree: an edge is part of the minimum spanning tree of a weighted graph $G$ if and only if there is no cycle in $G$ for which it is the longest edge. By adding edges from $x_{n+1}$ to $N$, we created at most $|N|$ new cycles. So for each newly created cycle, delete the longest weight edge in the cycle. Doing this, we delete at most $|N|$ edges. After this process, the remaining graph is exactly $M S T_{n+1}$, due to the characterization given above. Therefore, the number of edges that were added or 
deleted is bounded above by $2 \operatorname{deg}\left(x_{n+1}\right)$. In any dimension, two edges incident to the same point in the Euclidean minimum spanning tree must define an angle of at least 60 degrees, (see, e.g., Lemma 4 of Aldous and Steele [3]). Thus the value $\operatorname{deg}\left(x_{n+1}\right)$ is bounded above by a constant in any dimension $d$.

We can now establish an almost increasing property of the height of the tree explaining Kruskal:

Lemma 5. Let $x_{1}, x_{2}, \ldots, x_{n+1}$ be any points (not necessarily random) in $[0,1]^{d}$. Let $H_{n}$ and $H_{n+1}$ be the heights of the tree explaining Kruskal's algorithm for the point sets $\mathcal{X}_{n}=\left\{x_{1}, \ldots, x_{n}\right\}$ and $\mathcal{X}_{n+1}=\left\{x_{1}, \ldots, x_{n+1}\right\}$, respectively. Then, there exists some non-negative constant $a \geq 0$ independent of $\mathcal{X}_{n+1}$ such that

$$
H_{n+1} \geq H_{n}-a \text {. }
$$

Proof. Let $M S T_{n}$ be the minimum spanning tree on the point set $\mathcal{X}_{n}$ and let $T_{n}$ be the tree explaining Kruskal's algorithm for this point set, and as usual $H_{n}$ its height. Let $h=H_{n}$ for short, and let $\pi$ be a path of length $h$ in the tree $T_{n}$. Each non-leaf vertex in the path $\pi$ corresponds to an edge in $M S T_{n}$. Label these edges $e_{1}, \ldots, e_{h}$ starting from the bottom of the tree. Now consider $M S T_{n+1}$. Not all of the edges $\left\{e_{i}, 1 \leq i \leq h\right\}$ are also also in $M S T_{n+1}$. Let $K \subseteq[0, h]$ be the index set for those edges also in $M S T_{n+1}: K=\left\{k \in[1, h]: e_{k} \in M S T_{n+1}\right\}$. The goal is to show that $\left\{e_{k}: k \in K\right\}$ all lie on a single path in the tree explaining Kruskal for $M S T_{n+1}$. From Lemma $4,|K| \geq h-2 \operatorname{deg}\left(x_{n+1}\right)$, and therefore if the edges are indeed on the same path in $T_{n+1}$, then the height of this tree is at least $H_{n}-a$ for $a>0$ a positive constant.

Let $w\left(e_{k}\right)$ be the weight of the edge $e_{k}$ and for any $k \in K$ define $C_{e_{k}}$ to be the connected component in the graph $G\left(\mathcal{X}_{n+1}, w\left(e_{k}\right)\right)$ which contains the edge $e_{k}$. We show the following fact: The component $C_{e_{k}}$ contains all "previous" edges, $\left\{e_{j}: j \in\right.$ $K, j \leq k\}$. It is easy to see that this fact implies that the edges $\left\{e_{k}: k \in K\right\}$ lie on the same path in the tree $T_{n+1}$, since they all grow out from the same component. And the proof of the fact is also fairly simple: By definition of the edges $\left\{e_{i}, 1 \leq i \leq h\right\}$, there is a connected component in $G\left(\mathcal{X}_{n}, w\left(e_{k}\right)\right)$ containing all edges $\left\{e_{j}: j \leq k\right\}$. When we add a point, $x_{n+1}$, the vertices that are connected by edges of weight less than $w\left(e_{k}\right)$ are still connected — but perhaps by a different path. Therefore, all edges $\left\{e_{j}: j \in K, j \leq k\right\}$ are in the component $C_{e_{k}}$.

Finally, we are ready to prove our main theorem:

Proof of Theorem 1. Let $\epsilon \in(0,1)$. Let $H_{N}$ be the height of the tree explaining Kruskal, for a Poisson point process on $[0,1]^{d}$ with rate $n(1-\epsilon)$. Then by Theorem 4 , there exists a constant $c>0$ such that $\mathbf{P}\left\{H_{N} \geq c n(1-\epsilon)\right\} \rightarrow 1$, as $n \rightarrow \infty$. Either $N$ is close enough to its mean, and then we can apply Lemma 5 , or it is far from the mean, but this happens only with low probability. More precisely, let $A(n, \epsilon)$ be the event that $n-2 \epsilon n \leq N \leq n$ and let $A(n, \epsilon)^{c}$ be its complement.

$$
\mathbf{P}\left\{H_{N} \geq c n(1-\epsilon)\right\} \leq \mathbf{P}\left\{A(n, \epsilon)^{c}\right\}+\mathbf{P}\left\{H_{N} \geq c n(1-\epsilon), A(n, \epsilon)\right\} .
$$

Now, if $A(n, \epsilon)$ holds then $H_{n} \geq H_{N}-a(n-N)$, by Lemma 5. Therefore,

$$
\mathbf{P}\left\{H_{N} \geq c n(1-\epsilon), A(n, \epsilon)\right\} \leq \mathbf{P}\left\{H_{n} \geq c n(1-\epsilon)-a(n-N), A(n, \epsilon)\right\} .
$$


Pick $\epsilon \in\left(0, \frac{c}{2(c+2 a)}\right)$. This guarantees that

$$
\mathbf{P}\left\{H_{n} \geq c n(1-\epsilon)-2 a n \epsilon\right\} \leq \mathbf{P}\left\{H_{n} \geq c n / 2\right\} .
$$

Finally, we need a bound on the probability that $N$ is far from its mean. By Chernoff's bound [9],

$$
\mathbf{P}\left\{A(n, \epsilon)^{c}\right\} \leq \mathbf{P}\{|N-E N| \geq \epsilon n\} \leq 2 e^{-\gamma_{\epsilon} n},
$$

where $\gamma_{\epsilon}=\epsilon-\ln (1-\epsilon)>0$. Putting everything together, for this range of $\epsilon$,

$$
\mathbf{P}\left\{H_{N} \geq c n(1-\epsilon)\right\} \leq \mathbf{P}\left\{A(n, \epsilon)^{c}\right\}+\mathbf{P}\left\{H_{n} \geq c n / 2\right\}
$$

And thus,

$$
\mathbf{P}\left\{H_{n} \geq c n / 2\right\} \geq \mathbf{P}\left\{H_{N} \geq c n(1-\epsilon)\right\}-2 e^{-\gamma_{\epsilon} n} .
$$

Letting $n \rightarrow \infty$ proves that $\mathbf{P}\left\{H_{n} \geq c n / 2\right\} \rightarrow 1$.

\section{Acknowledgement}

We thank Michael Drmota for directing us towards this problem, and two anonymous referees for their helpful comments.

\section{References}

[1] A.V. Aho, J.E. Hopcroft, and J.D. Ullman. The Design and Analysis of Computer Algorithms. Addison-Wesley, Boston, MA, 1974.

[2] D. Aldous. A random tree model associated with random graphs. Random Structures and Algorithms, 4:383-402, 1990.

[3] D. Aldous and J. M. Steele. Asymptotics for euclidean minimum spanning trees on random points. Probability Theory and Related Fields, 92:247-258, 1992.

[4] B. Bollobás. Random Graphs. Cambridge Studies in Advanced Mathematics. Cambridge University Press, second edition, 2001.

[5] B. Bollobás and I. Simon. On the expected behavior of disjoint set union algorithms. In STOC '85: Proceedings of the seventeenth annual ACM Symposium on Theory of Computing, pages 224-231, New York, NY, USA, 1985. ACM.

[6] B. Bollobás and I. Simon. Probabilistic analysis of disjoint set union algorithms. SIAM Journal on Computing, 22:153-1074, 1993.

[7] C. Borgs, J.T. Chayes, H. Kesten, and J. Spencer. The birth of the infinite cluster: Finite-size scaling in percolation. Commun. Math. Phys., 224:153-204, 2001.

[8] O. Borůvka. O jistém problému minimálním. Práce Mor. Př írodověd. Spol. v Brně (Acta Societ. Scient. Natur. Moravicae), 3:37-58, 1926. 
[9] H. Chernoff. A measure of asymptotic efficiency for tests of a hypothesis based on the sum of observations. Annals of Mathematical Statistics, 23:493-507, 1952 .

[10] E. Dijkstra. A note on two problems in connection with graphs. Numer. Math., 1:269-271, 1959

[11] P. Erdős and A. Rényi. On the evolution of random graphs. Publ. Math. Inst. Hungar. Acad. Sci., 5:17-61, 1960.

[12] A. M. Frieze. On the value of a random minimum spanning tree problem. Discrete Applied Mathematics, 10:47-56, 1985.

[13] G. R. Grimmett. Percolation, volume 321 of A Series of Comprehensive Studies in Mathematics. Springer Verlag, second edition, 1999.

[14] S. Janson, T. Łuczak, and A. Ruciński. Random Graphs. Wiley, New York, 2000 .

[15] V. Jarńik. O jistém problému minimálnim. Práce Mor. Přírodověd. Spol. v Brně (Acta Societ. Scient. Natur. Moravicae), 6:57-63, 1930.

[16] H. Kesten. Percolation Theory for Mathematicians. Birkhäuser, Boston, 1980.

[17] D. E. Knuth and A. Schönhage. The expected linearity of a simple equivalence algorithm. Theoretical Computer Science, 6:281-315, 1978.

[18] J. B. Kruskal. On the shortest spanning subtree of a graph and the traveling salesman problem. Proceedings of the American Mathematical Society, 2:48-50, 1956 .

[19] C. McDiarmid, Th. Johnson, and H. S. Stone. On finding a minimum spanning tree in a network with random weights. Random Structures and Algorithms, 10 (1-2):187-204, 1997.

[20] R. Meester and R. Roy. Continuum Percolation. Cambridge Tracts in Mathematics. Cambridge University Press, 1996.

[21] M. Penrose. Random Geometric Graphs. Oxford Studies in Probability. Oxford University Press, 2003.

[22] M. Penrose. The longest edge of a random minimal spanning tree. The Annals of Applied Probability, 7(2):340-361, 1997.

[23] M. Penrose. Random minimal spanning tree and percolation on the n-cube. Random Structures and Algorithms, 12:63-82, 1998.

[24] M. Penrose. A strong law for the longest edge of the minimal spanning tree. The Annals of Probability, 27(1):246-260, 1999.

[25] R. C. Prim. Shortest connection networks and some generalizations. Bell Syst. Tech. J., 36:1389-1401, 1957. 
[26] J. M. Steele. Probability Theory and Combinatorial Optimization. CBMS-NSF Regional Conference Series in Applied Mathematics. SIAM, Philadelphia, 1997.

[27] A.C. Yao. On the average behavior of set merging algorithms. In STOC '76: Proceedings of the 8th Annual ACM Symposium on Theory of Computing, pages 192-195, 1976.

[28] J. E. Yukich. Probability Theory of Classical Euclidean Optimization Problems, volume 1675 of Lecture Notes in Mathematics. Springer Verlag, 1998. 